\title{
Le Riou Mort, affluent du Lot, pollué par métaux lourds. IV. Etude des Oligochètes.
}

\author{
N. Gianj1
}

Mots clés : Oligochaeta, écologie, eaux courantes, pollution.

La réponse des communautés d'Oligochète aux fortes pollutions domestiques et industrielles d'un petit ruisseau, le Riou Mort, est examinée. L'installation d'une communauté caractéristique (Tubifex iubifex, Limnodrilus hoffmeisteri, L. udekemianus, L. claparedeanus, Nais elinguis) s'accompagne d'une réduction du nombre d'espèces, d'une très forte augmentation de la densité et d'une uniformisation des peuplements entre les faciès lotique et lénitique.

La validité de certains indices structuraux (de diversité ou de dominance) appliqués aux seuls oligơhètes et de quelques paramètres spécifiques de ce taxon est discutee. Dans le cas du Riou Mort, il apparaît que des paramètres simples (densité, nombre d'espèces, proportion des diverses familles etc...) reflètent suffisamment bien les conditions de milieu et les Oligochètes sont utiles pour hiérarchiser les fortes pollutions.

La tolérance de Lumbricillus rivalis, Pristina idrensis et Trichodrilus allobrogum est à souligner.

The Rlou Mort, a tributary of the River Lot, polluted by heavy metals. IV. A study of the oligochaetes.

Keywords : Oligochaeta, ecology, running water, pollution.

The response of oligochaete communities to heary domestic and industrial pollution in a small stream, the Riou Mort. was investigated. The presence of a characteristic community (Tubifex ubifex, Limnodrilus hoffmeisteri, L. udekemianus, L. claparedeanus, Nais elinguis) was accompanied by a reduction in the number of species, by a marked increase in density, and by a standardisation of populations between the lotic and lenitic aspects.

The validation of certain st ructural indices (of diversity or dominance) applied to only the oligochaetes and of seve ral specific parameters for this group is discussed. In the ca se of the Riou Mort, it is apparent that simple parameters (density, number of species, proportion of diverse families, etc.) are an adequate indication of the environmental conditions. and the oligochaetes are useful for classifying different levels of severe pollution.

The tolerance of Lumbricillus rivalis, Pristina idrensis and Trichodrilus allobrogum is emphasised.

Le Riou Mort a déjà fait l'objet de plusieurs études consacrées à la physico-chimie et aux communautés algales (Say 1978), à l'accumulation des métaux lourds par les invertébrés (Say \& Giani 1981) et aux peuplements de macro-invertébrés benthiques (Giani 1983). Nous proposons ici une étude détaillée de la réponse des communautés d'Oljgochètes aux fortes pollutions caractéristiques de ce cours d'eau. Les résultats permettent de discuter la validité de différents critères, utilisés pour caractériser ces pollutions, lorsqu'ils sont appliqués aux seuls Oligochètes.

I. Laboratoire d'Hydrobiologie, UA 695 du CNRS, Université Paul Sabatier, 118, route de Narbonne, 31062 Toulouse Cedex FRANCE

\section{1. - Milieu et méthodes}

Le Riou Mort est un petit affluent du Lot qui draine le bassin houiller de Decazeville dans le département de l'Aveyron. Les stations étudiées ici (fig. l) ont été décrites précédemment (Giani 1983) : nous les caractériserons donc très brièvement.

Station 1 : station de référence : pas de pollution notoire.

Station 2 : à l'ent rée de Firmi ; polluée par les premiers égouts de cette ville.

Station 3 : en amont des aciéries de Decazeville; pollution organique par les égouts de Firmi et Decazeville.

Station 4 : à la sortie de Decazeville ; pollution organique et par les eaux usées des aciéries.

Station 5 : dans Viviez; identique à la précédente. 


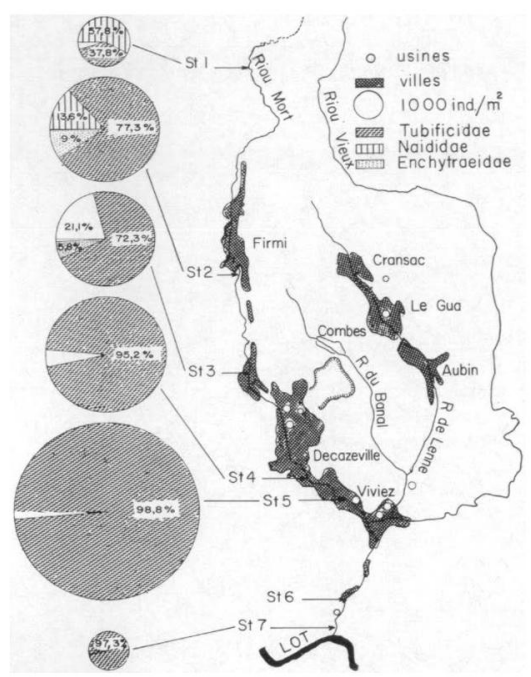

Fig. 1. Localisation des stations d'études et des principales sources de pollution. Les cercles indiquent les densités d'Oligochètes aux diverses stations.

Station 6 : en aval de la confluence avec le Riou Vieux ; pollution organique plus effluents d'une fonderie de zinc, de diverses usines métallurgiques et d'une fabrique de peinture.

Station 7 : après le rejet des eaux usées d'une centrale thermique.

Les paramètres physiconchimiques ont été décrits précédemment (loc. cit.) et ils peuvent se résumer ainsi :- débit d'étiage constitué à $90 \%$ par des rejets d'eaux usées industrielles et domestiques (S.O.D.E.T.E.G. 1976) :

- forte teneur en matière organique du sédiment (12 a $20 \%$ ) au niveau des stations 2 à 5 polluées organiquement :

- forte turbidité de l'eau à partir de la station 4 ;

- brusque élévation de température en 4 :

- augmentation brutale de la conductivité en 4 ;

- brutales variations du $\mathrm{pH}$ et de la teneur en $\mathrm{O}_{2}$ en aval du Riou Vieux en fonction des déversements des usines.

Six séries de prélèvements de faune ont été effectuées sur un cycle de un an, aux dates suivantes:05/08. 15/10, et $13 / 12$ en $1976 ; 22 / 02,18 / 04$ et $15 / 06$ en 1977 . A chaque station, 5 relevés ont été réalisés lors de chaque sortie : 2 au filet de Surber $\left(500 \mathrm{~cm}^{2}\right.$, vide de maille $\left.300 \mu \mathrm{m}\right)$ dans les zones en courant; 3 à l'aide de tubes en altuglass (diamètre : $8 \mathrm{~cm}$ ) dans les dépôts en courant lent).

\section{2. - Le peuplement d'Oligochètes}

Les Oligochètes représentent $77,5 \%$ du total des invertébrés récoltés. Le nombre d'individus de chaque espèce récoltée aux diverses stations figure dans le tableau I.

\section{1. - Répartition spatiale (fig. 2)}

- LUMBRICULIDAE : groupe de faible importance numérique, il constitue seulement $0,23 \%$ du total des Oligochètes. Il intervient toujours pour moins de $0,5 \%$ du peuplement à chaque station sauf en 7 $(1,36 \%)$. Stylodrilus heringianus a surtout été récoltée, en faible quantité (densité maximale de 94 ind $/ \mathrm{m}^{2}$ en juin), à la station 1 non polluée. Lumbriculus variegatus, tolérante aux pollutions organiques, est présente aux stations $3,4,5$ et 7 avec une abondance maximale en 3 où la densité est cependant faible : 224 ind. $/ \mathrm{m}^{2}$ au maximum, en août. Tri. chodrilus allobrogum peuple exclusivement, mais faiblement, les stations 4 et 5 avec une densité maxi. male de 3627 ind. $/ \mathrm{m}^{2}$ en février (st. 4). A notre con-

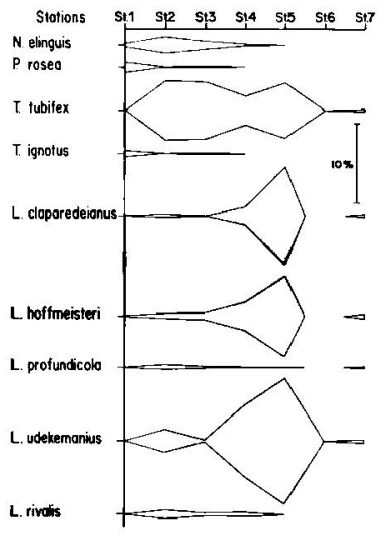

Fig. 2. Répartition longitudinale des principales espèces d'Oligochètes. 
Tableau I. Tableau récapitulatif des nombres d'Oligochètes récoltés aux diverses stations.

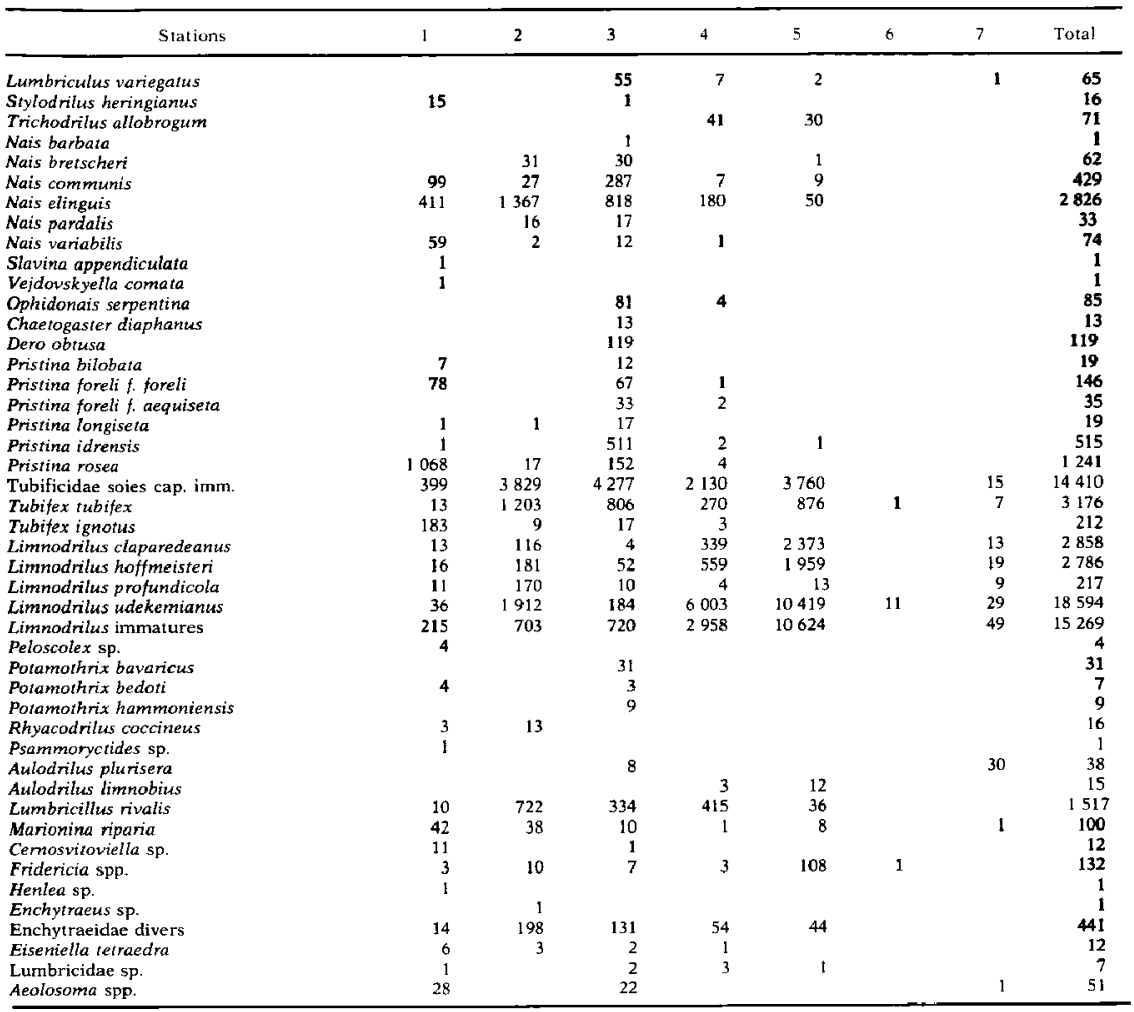

naissance, cette espèce n'avait jamais été récoltée dans des milieux aussi pollués.

- NAIDIDAE : avec 17 espèces, cette famille qui ne regroupe que $7,3 \%$ des Oligochètes est la plus diversifiée. Ils sont absents aux stations 6 et 7 ; ils représentent $57,8 \%$ des Oligochètes à la station 1 et moins de $1,5 \%$ en 4 et 5 ; $95 \%$ ont été récoltés en 1,2 et 3 . Leur répartition reflète bien les diverses agressions subies par le milieu et témoigne de la sensibilité de ce groupe aux pollutions non organiques et d'une certaine tolérance aux pollutions organiques.
4 espèces (Nais communis, $N$. elinguis, Pristina idrensis et $P$. rosea) forment $89,6 \%$ des Naididae dont $52,9 \%$ pour la seule $N$. elinguis. Certaines espèces sont bien représentées aux stations 1 et 3 et moins bien en 2 ; cette répartition résulte de la forte pollution organique de la station 2 suivie d'une amélioration en 3. Ce sont: $N$. communis (maximum d'abondance plus marqué en 3 ), $N$. variabilis et $P$. rosea (maximum plus marqué en 1) et $P$. foreli. Mais $N$. variabilis et $P$. foreli interviennent pour moins de $0,56 \%, P$. rosea pour $1,47 \%$ et $N$. communis pour $2,8 \%$ du peuplement de la station 3 compte tenu de 
la prolifération des Tubificidae. Les densités maximales observées peuvent être élevées : $N$. communis : 4697 ind. $/ \mathrm{m}^{2}$ en décembre à la station $2 ; N$. variabilis : 372 ind $/ \mathrm{m}^{2}$ en octobre en $1 ; P$. foreli : 1576 ind $/ \mathrm{m}^{2}$ en actobre en $1 ; P$. rosea : 23606 ind. $\mathrm{m}^{2}$ en octobre en 1 . Une certaine tolérance de $N$. communis à la pollution avait déjà été signalée par Bennike \& Berg (1948), Szczesny (1974) et Lafont (1977a). N. variabilis a été récoltée sur des filtres d'épuration par Learner (1979). N. elinguis a, au contraire, une abondance maximale à la station 2. Cette forme polluorésistante (Brinkhurst 1965, Ladle 1971. Kasprzak 1971, Lafont 1977 a et b) est abondante dans les milieux pollués, riches en Cladophora (Brinkhurst 1962), en mycéliums (Nuttal 1973) et sur les filtres d'épuration (Learner 1979). Elle est considérée comme un indicateur de pollution ou d'eutrophisation ( $\alpha, \beta$ mésosaprobe, mésotrophe) par Biesadka \& Kasprzak (1977), Howmiller \& Scott (1977), Dumnicka (1978), Dumnicka \& Pasternak (1978)... La densité maximale observée (8699 ind $\left./ \mathrm{m}^{2}\right)$ est faible comparée à celles relevées par Eyres et al (1978) : $59130 \mathrm{ind} . \mathrm{m}^{2}$, Dumnicka (1978) : $189000 \mathrm{ind} . / \mathrm{m}^{2}$, Szczesny (1974) : $200000 \mathrm{ind} . / \mathrm{m}^{2} .99 \%$ des individus de $P$. idrensis ont été récoltés en 3 avec une densité maximale de 1779 ind. $/ \mathrm{m}^{2}$ en octobre. Elle est abondante sur les filtres des stations d'épuration (Learner 1979). La polluorésistance de cet te espèce, observée ici, n'a été que très rarement soulignée : Learner et a! (197I), Eyres \& al (1978).

- TuBificidae : les 14 espèces recensées représentent $89 \%$ des Oligochètes. Tubifex tubifex, Limnodrilus hoffmeisteri, $L$. claparedeanus et $L$. udekemianus forment $96,7 \%$ du total des Tubificidae ; si l'on ajoute $T$. ignotus et $L$. profundicola ce nouveau groupe représente $99,3 \%$ de ce total. Les Tubificidae sont absents en 6 ; les autres stations peuvent se répartir en 3 groupes; station $1: 37,8 \%$ de Tubificidae, stations 2 et $3: 77,8 \%$ et $72,2 \%$, stations 4,5 et $7: 95,8 \%, 93$ et $97,3 \%$. L'abondance relative des Tubificidae traduit bien l'aggravation de la pollution aux diverses stations.

Peloscolex sp. (119 ind $/ \mathrm{m}^{2}$ maximum, en août) et Psammoryctides sp. (524 ind $/ / \mathrm{m}^{2}$ en octobre) ne sont présents qu'à la station 1 ; Potamothrix hammoniensis et $P$. bavaricus ont été récoltés à la station 3 uniquement ; $P$. bavaricus représente $2,1 \%$ du peuplement et sa densité maximale est de 1075 ind.$/ \mathrm{m}^{2}$ en août ; $P$. hammoniensis est moins abondante ; $0,6 \%$ et $76 \mathrm{ind} . / \mathrm{m}^{2}$ en octobre. Aulodrilus pluriseta, sur- tout récoltée à la station 7 , est également une espèce secondaire $(0,055 \%$ du peuplement $)$.

Parmi les six principales espèces de Tubificidae, seul T. ignotus (1,03\% des Oligochètes) présente une abondance maximale en 1 où sa densité maximale est de $2773 \mathrm{ind} / \mathrm{m}^{2}$ en décembre. Aux stations 2,3 et $4 \mathrm{il} \mathrm{représente} \mathrm{moins} \mathrm{de} 1,5 \%$ des Oligochètes. Cette espèce ne supporte pas la pollution (Dumnicka \& Pasternak 1978) bien que comme Kasprzak (1976) et Szczesny (1974) nous l'ayons récoltée en petit nombre dans les stations polluées. Milbrink (1980 et 1983) associe cependant cet te espece aux pollutions.

L. protundicola (1,3\% des Oligochètes) a une abondance maximale en 2 où il représente $7,01 \%$ du peuplement. Il forme $4,6 \%$ des Oligochètes en 1 et $2,3 \%$ en 3 et moins de $0,15 \%$ en 4 et 5 . La présence de cette espèce classée parmi les forces caractéristiques des lacs Oligotrophes (Howmiller \& Scott 1977, Milbrink 1980 et 1983) - ce qui est contesté par Lafont \& Juget (1981) - confirme les observations de Dumnicka \& Pasternak (1978) et de Dumnicka (1978) pour qui $L$ profundicola peut supporter une pollution modérée.

T. uubifex est, numériquement, le deuxième Oljgochète récolté $(25,8 \%)$. Il atteint son abondance maximale aux stations 2,3 et 5 où il représente respectivement $34 \%, 51.4 \%$ t $15.9 \%$ des Oligochètes ; en 1 et 4 il forme cependant 12,1 et $11 \%$ du peuplement. Bien connu pour sa polluorésistance il est souvent considéré comme polysaprobe et indicateur d'eutrophie. Il peut être abondant dans les milieux oligotrophes (Juget \& Giani 1974). En fait, il peut survivre dans des conditions extrêmes si la compétition est limitée (Brinkhurst 1969) ce qui explique la dualité écologique de cette espèce (Milbrink 1980 et 1983 ). Le peuplement ne dépasse pas $2685 \mathrm{ind} / \mathrm{m}^{2}$ en 1 (août) alors qu'il peut atteindre 48755 ind. $/ \mathrm{m}^{2}$ en 4 (août). Ces chiffres sont faibles comparés à celui de Mc Lusky \& al (1980) par exemple : 127400 ind. $/ \mathrm{m}^{2}$.

L. claparedeanus $(15,6 \%$ des Oligochètes) représente $4,72 \%$ du peuplement en 1, 4,27\% en 2 . $0,94 \%$ en $3,9,4 \%$ en 4 et $18,8 \%$ en 5 . Les densités maximales reflètent bien le degré de pollution : 2292 ind. $/ \mathrm{m}^{2}$ en août à la station 1,3280 ind. $/ \mathrm{m}^{2}$ en 2 (octobre), 514 ind. $\mathrm{m}^{2}$ en 3 (août), 42310 ind. $/ \mathrm{m}^{2}$ en 4 (août), 74114 ind $/ \mathrm{m}^{2}$ en 5 (octobre). Cette espèce polluorésistante est considérée comme un indicateur d'eutrophie (Howmiller \& Scott 1977, Milbrink 1980 et 1983). 
L. hoffmeisteri (16,8\% des Oligochètes) a un développement maximal en 4 et 5 mais sa contribution au peuplement croît régulièrement à chaque station : $5,4 \%$ en $1,6,9 \%$ en $2,13,4 \%$ en $3,18,2 \%$ en 4 et $19,2 \%$ en 5 . Les densités maximales varient de 2340 ind. $/ \mathrm{m}^{2}$ en 1 (août) à 92293 ind. $/ \mathrm{m}^{2}$ en 4 (août). II est considéré comme polysaprobe (Brinkhurst 1962...) et eutrophe (Howmiller \& Scott 1977, Milbrink 1983...). Selon Chapman \& al (1982) il est le plus cosmopolite et le plus utile des indicateurs de pollution. Brinkhurst (1966 et 1967) admet que le pourcentage de $L$. hoftmeisteri dans la communauté d'Oligochètes est un bon critère de pollution organique.

L. udekemianus est la forme dominante dans le Riou Mort $(28,5 \%)$. Il intervient pour 1,57\% du peuplement en $1,28,8 \%$ en $2,5,3 \%$ en $3,59,2 \%$ en 4 et $44,9 \%$ en 5 . Les densités maximales varient de 704 ind. $/ \mathrm{m}^{2}$ en 1 (juin) à 359394 ind. $/ \mathrm{m}^{2}$ en 4 (août). Selon Brinkhurst (1962 et 1965) cette espèce est modérément polluorésistante ; selon Kennedy (1965) et Lafont (1977a) elle n'est pas très abondante en milieu pollué. Mais Gross (1976), Dumnicka \& Pasternak (1978), Eyres \& al (1978) la considerent comme très polluorésistante et abondante en milieu pollué. Dumnicka (1978) la qualifie de polysaprobe ce qui correspond bien à nos observations.

En résumé, parmi les Tubificidae du Riou Mort nous pouvons distinguer 4 types de réponse à la pollution : régression brutale pour $T$. ignotus; $L$. profuncicola est favorisée par une pollution moyenne, au delà elle régresse ; $L$. hoffmeisteri, L. claparedeanus et L. udekemianus progressent au fur et à mesure que la pollution augmente ; T. tubifex progresse brutalement dès la première station polluée et se maintient ensuite au même niveau.

- Enchytraeidae : ils représentent $3,3 \%$ des récoltes. Seulement 6 genres ou espèces ont pu être recensés compte tenu des difficultés de détermination du matériel fixé. Ils sont bien représentés aux stations 2,3 et 4 , polluées. Ils forment $2,7 \%$ du peuplement en $1,9,05 \%$ en $2,5,8 \%$ en $3,3,13 \%$ et 4 et seulement $0,5 \%$ en 5 . Lumbricillus rivalis $(2,1 \%$ des Oligochètes) bien représenté en 3 et 4 atteint son abondance maximale en 2 . La densité maximale est de 4580 ind. $/ \mathrm{m}^{2}$ en 2 (décembre). La tolérance aux pollutions organiques de $L$. rivalis a été signalée par Hawkes (1962) et Learner \& al (1971) mais n'a pas retenu l'attention car, selon Brinkhurst (1966), les Enchytraeidae sont difficiles à déterminer et auraient une faible signification dans les études de pollution. Selon Learner \& al (1971), L, rivalis semble réagir comme les Tubificidae. Il a été récolté sur les filtres d'épuration (Williams \& Taylor 1968, Williams et al 1969); Martinez-Ansemil (1982), Dumnicka \& Pasternak (1978) l'ont trouvé dans des milieux très pollués. En accord avec Dumnicka (1978) nous le considérons comme polysaprobe.

Nous avons récolté Marionina riparia aux stations $1,2,3,4,5$ et 7 avec une abondance maximale en 2. Ceci confirme la polluorésistance de cette espèce (Dumnicka 1978, Lafont 1982).

- LUMBricidAE : groupe mineur $(0,025 \%$ des Oligochètes) il intervient pour moins de $0,2 \%$ du peuplement de chaque station. La densité maximale est de 177 ind. $/ \mathrm{m}^{2}$ aux stations 3 (juin) et 4 (février).

- Aeolosomatidae : nous les assimilons aux Oligochètes dont ils ne constituent que $0,075 \%$ des récol tes; mais ils représentent $0,05 \%$ du peuplement de la station 3 , fortement polluee, où leur densité maximale est de $1095 \mathrm{ind} / \mathrm{m}^{2}$ (octobre). Eyres $\&$ al (1978) ont souligné l'association Aeolosoma beddardi - P. idrensis dans un milieu très pollué. A la station 3 les Aeolosomatidae sont aussi associés avec P. idrensis.

\section{2. - Evolution saisonnière des populations}

$N$. elinguis présente une abondance maximale de février à avril (fig. 3) conformément à nos observations précédentes (Giani \& Martinez-Ansemil 1981). Selon les stations, 1,5 à $6,5 \%$ des individus sont matures en avril et jusqu'à $25 \%$ en juin. Ce dernier chiffre est supérieur à ce que nous avions observé précédemment (loc. cit.) et il correspond aux données de Learner \& al (1978).

N. communis (fig. 3), moins abondante que l'espèce précédente, et totalement absente en été (août) ; elle présen te deux maxima : à la fin du printemps (juin) et en hiver (décembre). Quelques individus sexués (2,6\% de la population) ont été récoltés à la station 3 en juin.

P. idrensis (fig. 3) n'a été récoltée qu'à la fin du printemps et au début de l'automne (oc tobre) où elle prolifère.

$P$. rosea (fig. 3) présente, comme P. idrensis, une abondance maximale en octobre ( $94 \%$ des individus) mais ne disparaît totalement qu'en été. Nous n'avons pas récolté de Pristina sexuées. 

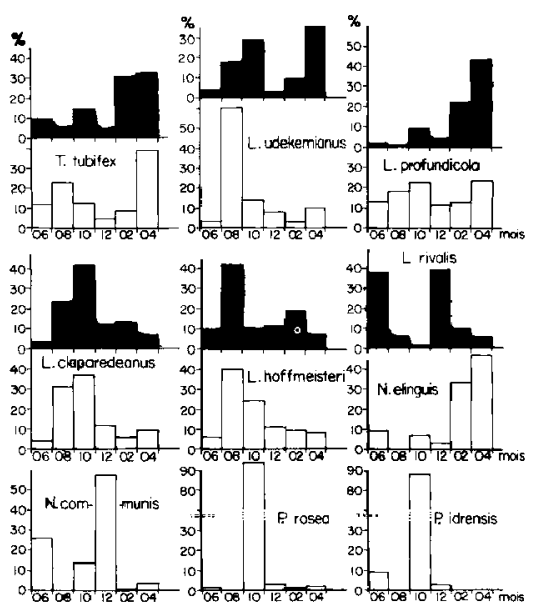

Fig. 3. Évolution des populations des principales especes d'Oligochètes aux diverses pédiodes de prélèvements (Pour T. rubifex, L. claparedeanus, L. hoffmeiszeri, L. profundicola et $L$. udekemianus l'histogramme noir représente l'évolution de la population sexuée).

Les Naididae sont presque totalement absents en été $(0,047 \%$ récoltés en août). Le reste de l'année chaque période est caractérisée par la prolifération d'une ou deux espèces. Ainsi, $39 \%$ ont été récoltés en octobre (essentiellement $P$, rosea et $P$. idrensis); $17 \%$ en février et $25,1 \%$ en avril (période d'abondance maximale de $N$. elinguis) : $9,9 \%$ en juin et $8,8 \%$ en décembre (maxima d'abondance de $N$. communis). De méme $80,8 \%$ des Naididae de la station 1 ont été récoltés en octobre ; $38 \%$ en avril pour la station $2 ; 36,9 \%$ en avril pour la station $3 ; 80,5 \%$ en février à la station 4 et 75,8\% en février à la station 5. Les Naididae colonisent les stations 4 et 5 en hiver seulement, lors de l'atténuation des pollutions par les hautes eaux et les basses températures.

La population de $T$. tubifex présente un minimum en décembre et deux maxima en août et surtout en avril. Nous avons observé des individus sexués toute l'année mais le nombre d'adultes est maximal en février et en avril et minimal en août et décembre (fig. 3). Le renouvellement de la population est sur- tout intense en avril et il se poursuit tout l'été. Ceci correspond aux observations de Brinkhurst \& Kennedy (1965), Ladle (1971), Gross (1976) et Eyres \& al (1978).

La population de L. hoffmeisteri est maximale en août et elle décroît ensuite progressivement (fig. 3). La proportion d'individus sexués est toujours très élevée ; elle est maximale en février où une forte tranche de la population arrive à l'âge adulte. Cependant, le nombre d'individus sexués est maximal en août, à la période de renouvellement maximal de la population conformément aux observations de Ladle (1971) et Eyres \& al (1978).

L'évolution des populations de $L$. claparedeanus (fig. 3) est identique à celle de $L$. hotfmeisteri mais avec un décalage de la phase de reproduction maximale vers la fin de l'été.

L. udekemianus a une évolution saisonnière ( $f$ ig. 3) en tout point comparable à celle de $L$. hotfmeisteri.

Les populations de L. profundicola sont relativement constantes dans le temps (fig. 3); deux légers maxima, correspondant aux phases de reproduction maximale, s'observent en octobre et en avril et le renouvellement est faible de février à juin, période où 68 à $82 \%$ d'individus sont adultes. T. ignotus présente deux maxima en juin et décembre, période où nous avons récolté des individus sexués : $10,8 \%$ de la population en juin et $16,5 \%$ en décembre.

Pour les Tubificidae on peut donc distinguer les espèces à période de reproduction bien délimitée dans le temps ( $T$. ignotus) et celles dont la reproduction s'effectue toute l'année avec des pics peu marqués (L. profundicola) ou très marqués (printemps : T. tubifex ; été : L. hoffmeisteri et L. udekemianus ; fin de l'été : $L$. claparedeanus). Les maxima de chaque espèce sont étalés du printemps à la fin de l'été ; les minima s'observent en automne et en hiver.

Les fluctuations saisonnières résultant soit du cycle biologique soit de l'amélioration temporaire du milieu influencent très fortement la structure du peuplement et doivent être prises en compte dans l'analyse des indices structuraux.

\section{Remarque : Le Parasitisme}

Les grandes densités observées permettent le développement d'une population d'un Cestode néoténique du gen re Archigetes sur des Limnodrilus. A la station 3,2 L. hoffmeisteri étaient parasités et de nombreux autres hébergeaient des œufs à clapet 
identiques à ceux d'A rchigetes. Aux stations suivantes ces Cestodes parasitent L. udekemianus. Nous avons observé 14 Archigetes en 4, 58 en 5 et un seul en 7. Ces individus, dont l'utérus était généralement rempli d'œufs, ont été observés indifféremment sur des spécimens matures ou immatures de L. udekemianus au niveau des segments génitaux (11 à 17); 2 ont cependant été observés sur des segments plus postérieurs (27 à 30 et 41 à 45). Généralement chaque hôte abritait un seul parasite mais deux individus hébergeaient chacun deux Cestodes. Moins de $1 \%$ des Limnodrilus étaient parasités sauf en avril à la station 5 où la proportion atteignait $12 \%$.

\section{3. - Caractéristiques faunistiques des diverses stations}

Chaque station peut-être caractérisée à partir de son peuplement en Oligochètes. Les principaux paramètres de ce peuplement sont regroupés dans le tableau II.

- Station 1 (fig. 4) : la forte proportion d'Oligochètes résulte d'une accumulation de sable. Le peuplement, diversifié, est dominé par les Naididae. 5 espèces n'ont été récoltées qu'à cette station mais en faible nombre ( $S$. appendiculata, $V$. comata, Peloscolex $\mathrm{sp}$. Psammoryctides sp. S. heringianus). Les especes dominantes sont : $P$. rosea $(35,8 \%)$. $T$. ignotus $(22,8 \%)$ et $N$. elinguis $(13,8 \%)$. N. variabilis $(1,97 \%)$ est une espèce secondaire.

- Station 2 (fig. 5) : la diversité diminue, les $\mathrm{Naj}$ didae régressent et les Enchytraeidae progressent ; les Tubificidae sont dominants. Aucune espèce n'a été récoltée exclusivement à cette station. Le peu-

Tableau II. Principales caractéristiques des peuplements d'Oligochètes aux diverses stations.

\begin{tabular}{lcccccc}
\hline \multicolumn{1}{c}{ Stations } & 1 & 2 & 3 & 4 & 5 & 7 \\
\cline { 2 - 7 } & & & & & & \\
$\begin{array}{l}\text { Pourcentage } \\
\text { d'Oligochètes } \\
\text { dans le peuple- }\end{array}$ & 67,3 & 46,8 & 62,6 & 98,6 & 99,8 & 60,4 \\
$\begin{array}{l}\text { ment } \\
\begin{array}{l}\text { Nombre de taxa } \\
\text { d'Oligochètes }\end{array}\end{array}$ & 30 & 20 & 35 & 22 & 18 & 9 \\
$\begin{array}{l}\text { Tubificidae } \\
\text { (en \%) }\end{array}$ & 37,8 & 77,3 & 72,3 & 95,2 & 98,8 & 97,3 \\
$\begin{array}{l}\text { Naididae (en \%) } \\
\text { Enchyt raeidae } \\
\text { (en \%) }\end{array}$ & 57,8 & 13,6 & 21,1 & 1,3 & 0,25 & 0 \\
\hline
\end{tabular}

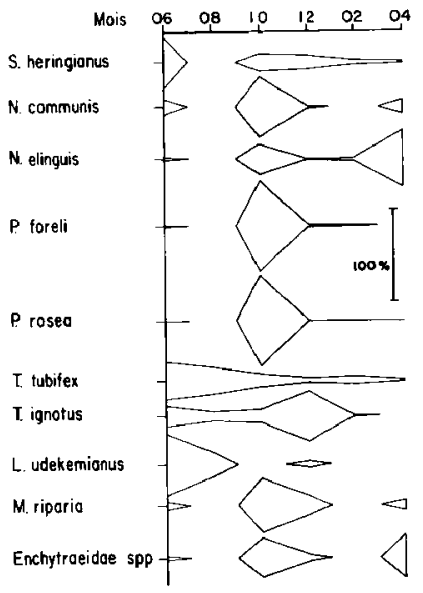

Fig. 4. Evolution des populations des principales espèces d'Qligochètes aux diverses dates de prélèvements à la station 1 .

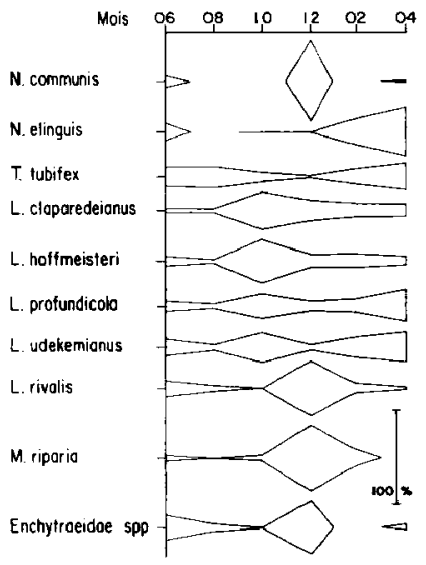

Fig. 5. Evolution des populations des principales espèces d'Oligochètes aux diverses dates de prélèvements à la station 2 . 


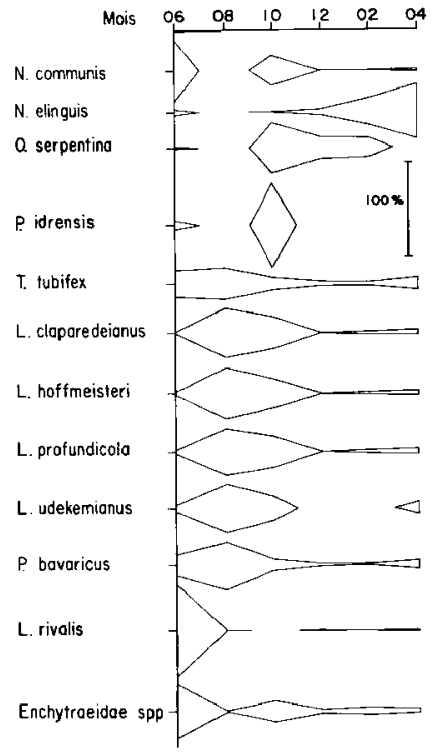

Fig. 6. Évolution des populations des principales espèces d'Oligochètes aux diverses dates de prélèvements à la station 3 .

plement est dominé par T. tubifex, $(47,1 \%)$, L. udekemianus $(18,2 \%)$ et $N$. elinguis $(12,8 \%)$. L. rivalis $(6,7 \%)$ et $L$. protundicola $(7,01 \%)$ sont les espèces secondaires. $N$. bretscheri et $N$. pardalis atteignent leur abondance maximum à cette station.

- Station 3 (fig. 6) : la progression des Naididae. le recul des Enchytraeidae reflètent l'amélioration de la qualité des eaux ; les Tubificidae avec $T$. tubifex $(56,5 \%)$ sont dominants. N. elinguis $(7,94 \%), L$. hoffmeisteri $(7,3 \%)$ et $P$. idrensis (5\%) sont des espèces secondaires. $P$. bavaricus $(2,17 \%)$ et $P$. hammoniensis $(0,6 \%)$ n'ont été récoltés qu'à cette station. $N$. communis $(2,8 \%)$ est également caractéristique.

La richesse spécifique à cette station s'explique par la légère récupération des eaux et par la présence d'espèces $(N$. barbata, $O$. serpentina, $C$. diaphanus, $D$. obtusa, $P$. aequiseta, A. pluriseta) probablement issues du deversoir d'un petit étang situé en amont comme nous l'avions déjà noté précédemment (Giani 1983). Certaines de ces espèces sont d'ailleurs connues pour vivre dans les eaux calmes et les herbiers.

- Station 4 (fig. 7) : le nombre de taxa diminue; les Tubificidae dominent très fortement ; les Naididae sont en forte régression; les Enchytraeidae se maintiennent. Aucune forme n"a été récoltée exclusivement à cette station. Seul, $T$. allobrogum $(0,27 \%)$ atteint son abondance maximale en 4. L. udekemianus $(48,6 \%)$, T. tubifex $(18,6 \%)$, L. hoffmeisteri $(18,5 \%)$ et $L$. claparedeanus $(11,3 \%)$ sont les espèces dominantes, $L$. rivalis $(2,75 \%)$ et $N$. elinguis $(1,2 \%)$ sont des formes secondaires. Les 16 taxa restants représentent chacun moins de $0,5 \%$ de la communauté.

- Station 5 (fig. 8) : le peuplement est formé presque en totalité par les Tubificidae. Aucune espèce n'a été capturée exclusivement à cette station mais il faut noter, comme en 4 , la présence de $T$. allobrogum. $L$. udekemianus $(34,4 \%)$, L. claparedeanus (26,9\%, L. hoffmeisteri (22,2\%) et T. tubifex (15,2\%) constituent la quasi totalité du peuplement légèrement dominé par L. udekemianus.

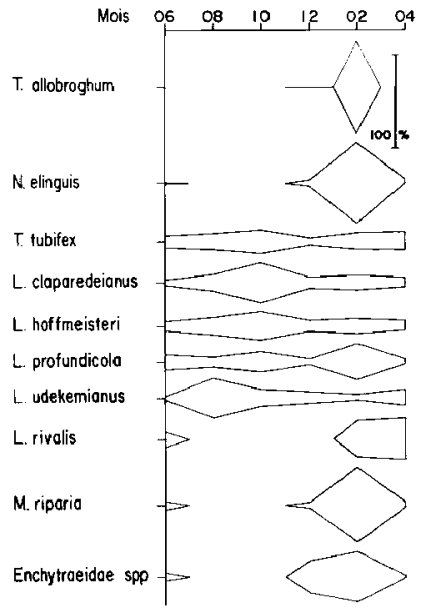

Fig. 7. Evolution des populations des principales espèces d'Oligochètes aux diverses dates de prélèvements à la station 4 


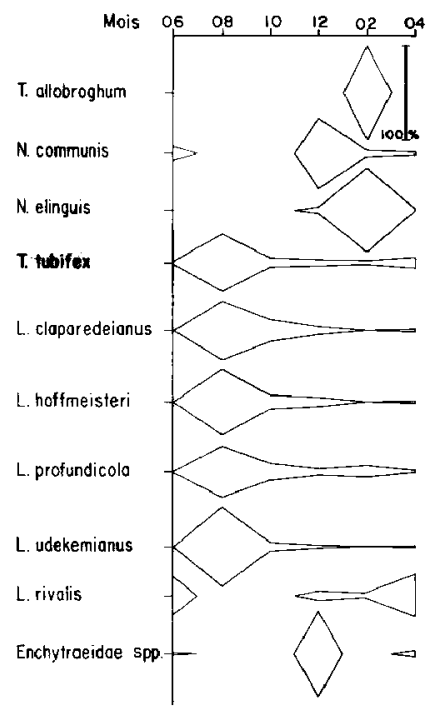

Fig. 8. Evolution des populations des principales espèces d'Oligochètes aux diverses dates de prélèvements à la station 5 .

- Station 6 : l'aggravation des pollutions d'origine industrielle à cette station entraîne une disparition totale de la faune, y compris les Oligochètes (Giani 1983).

-. Station 7 : Les Tubificidae (97,3\%) sont dominants ; les Lumbriculidae (L. variegatus) représentent $1,36 \%$ de la communauté. Seul $A$. pluriseta ( $2 \%)$ atteint son maximum d'abondance en 7 ; il recolonise, probablement depuis le Lot, les sédiments du Riou Mort. T. tubifex et les 4 Limnodrilus forment chacun de 12 à $20 \%$ de la communauté ; aucune espèce ne domine. Il s'agit d'un peuplement de recolonisation, fluctuant dans le temps (Giani 1983).

\section{3. - Etude de quelques paramètres du peuplement}

\section{1. - Nombre de taxa présents à chaque station}

Le nombre moyen ou total de taxa d'Oligochè à chaque station ( $f i g .9 \mathrm{~A}$ )varie de façon identique à celui de l'ensemble de la faune (Giani 1983). Il diminue après chacune des pollutions (stations 2, 4 et 6 ) et il progresse après chaque récupération (stations 3 et 7). L'évolution saisonnière du nombre de taxa à chaque station (fig. 9B) montre que le peuplement est riche en automne et, à un degré moindre au printemps; il est très appauvri en été.

\section{2. - Densité des populations d'Oligochètes}

La densité moyenne évolue comme le nombre de taxa mais en sens inverse comme cela a été souvent souligné (Woodiwiss 1964, Dratnal \& Kasprzak $1980 \ldots$..) Elle est pourtant maximale en été (août à octobre) sauf en 2 où elle va de pair avec une prolifération de $N$. elinguis en avril (fig. 9C). Eyres \& al (1978) ont également montré une plus grande abondance des mêmes espèces de Tubificadae, dans une rivière polluée, en été lorsque la pollution est la plus forte.

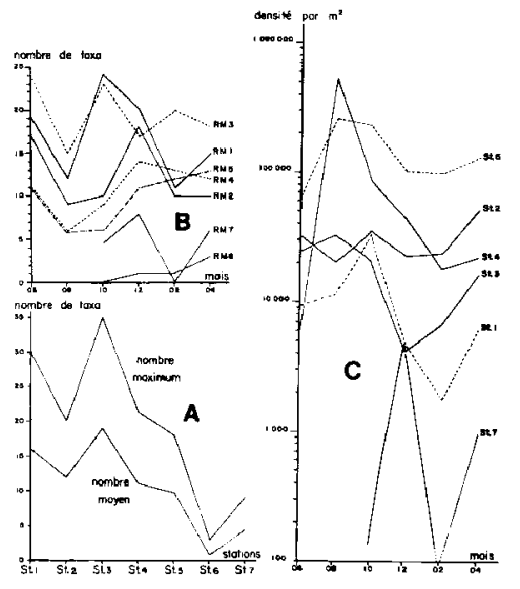

Fig. 9. Evolution de quelques paramètres du peuplement : $A$ : évolution du nombre de taxa présents aux diver. ses stations; $B$ : évolution annuelle du nombre de taxa à chaque station ; $C$ : évolution annuelle de la densité d'Oligochè tes à chaque station. 


\section{3. - Structure du peuplement}

Afin de déterminer la structure du peuplement nous avons calculé, sur les valeurs moyennes annuelles quelques indices structuraux couramment utilisés (cf. Hellawell 1978) : l'indice de diversité $\left(\mathrm{H}^{\prime}\right)^{l}$ de Shannon et Weaver et son complément $\left(\mathrm{J}^{\prime}\right)$ l'indice de diversité relative (evenness de Pielou 1975), l'indice de dominance (1-q) de Simpson (1949) ainsi que les paramètres proposés par Bournaud \& Keck (1980). Les résultats sont regroupés dans le tableau III.

Tableau III. Valeurs, calculées sur les moyennes annuelles à chaque station, de l'indice de diversité de Shannon \& Weaver ( $\left.\mathrm{H}^{\prime}\right)$ de l'indice de diversité relative ( $\left.J^{\prime}\right)$ de l'indice de dominance de Simpton (1-q) ainsi que des paramètres proposées par Bournaud \& Keck: moyenne (x) et moment d'ordre $3\left(\mathbf{M}_{3}\right)$. ( $\mathbf{P i}$ : fréquence relative de l'espèce $\mathrm{i}, \mathrm{S}$ : nombre de taxa, $\mathrm{x}$ : cote de la classe (voir figure 11), Sx : nombre de taxa dans la classe de cote $x$ ).

\begin{tabular}{|c|c|c|c|c|c|}
\hline \multirow[b]{2}{*}{ Stations } & $\begin{array}{c}\mathbf{H}^{\prime}= \\
2 \mathbf{P i} \log _{2} \mathbf{P i}\end{array}$ & $\begin{array}{l}\mathbf{y}^{\prime}= \\
\underline{H^{\prime}}\end{array}$ & $\begin{array}{c}1-\mathrm{q} \\
\mathrm{q}=\mathrm{zPi}^{2}\end{array}$ & $\begin{array}{l}\bar{x}= \\
\text { Sx X } \\
\end{array}$ & $\begin{array}{l}M_{3}= \\
(x-x) \\
\end{array}$ \\
\hline & & $\overline{\log }_{2}$ & & $\mathrm{~s}$ & s \\
\hline St. 1 & 2,96 & 0.605 & 0,794 & 3,6 & 7,91 \\
\hline St. 2 & 2,42 & 0,559 & .719 & 5,25 & 12,6 \\
\hline St. 3 & 2,69 & 0.524 & & 4,66 & 8,73 \\
\hline St. 4 & 2,09 & 469 & 0.7 & 3,95 & 35,61 \\
\hline St. 5 & 2,06 & 0.494 & 0,737 & 5,17 & 50,9 \\
\hline S1. 7 & 2,52 & 0,795 & 0,803 & 5,55 & $-0,31$ \\
\hline
\end{tabular}

Les valeurs de 1 - $q$ aux stations 4,5 et 7 traduisent une diminution de la dominance et donc de la pollution, ce qui est contraire à la réalité.

Les faibles variations de $\mathrm{H}^{\prime}(f \mathrm{~g}$. $10 \mathrm{~A})$ reflètent les conditions de milieu sauf en 5 où la baisse de diversité est compensée par une augmentation de l"équitabilité. Il est nécessaire de faire la part entre la diversité due à la répartition des effectifs entre les taxa et celle due à la richesse spécifique (Dills \& Rogers 1974, Ghetti \& Bonazzi 1977, Hellawell 1978, Bournaud \& Keck 1980). Selon Wilhm (1970) $\mathrm{H}^{*}$ comprix entre 3 et 4 indique une bonne qualité des eaux, en dessous de 1 il traduit une forte pollution. Ceci ne correspond pas à nos résultats et cet indice doit être utilisé de façon relative.

1. $\mathrm{H}^{\prime}, \mathbf{x}$ et $\mathbf{M}_{3}$ ont été calculés sur micro-ordinateur à l'aide de programmes élaborés par A. DAUTA que nous tenons à remercier.

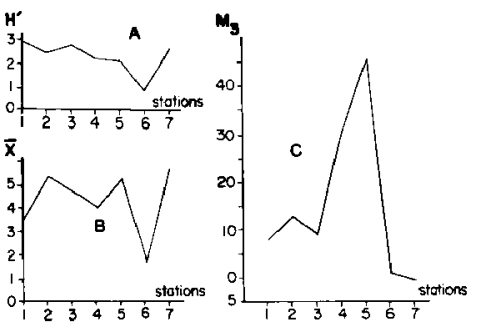

Fig. 10. Evolution de quelques indices structuraux à chacune des stations. A : indice de Shannon \& Weaver $\left(H^{\prime}\right)$. B : cote moyenne d'abondance $(x)$. C : moment d'ordre $3\left(\mathbf{M}_{3}\right)$ des distributions des cotes d'abondance (x et $\mathrm{M}_{3}$ selon Bournaud \& Keck 1980).

L'évolution de J' est comparable et elle ne traduit pas correctement l'évolution des conditions de milieu en 3 et 5 .

La distribution abondance-espèce (fig. 11 ) illustre bien l'évolution de la structure des communautés. En 1, le peuplement est diversifié ; les classes à effectif faibles (1 à 3) ou moyens (4 à 6) dominent. En 2, les classes à effectifs forts $(\geqslant 7)$ progressent. En 3 ,
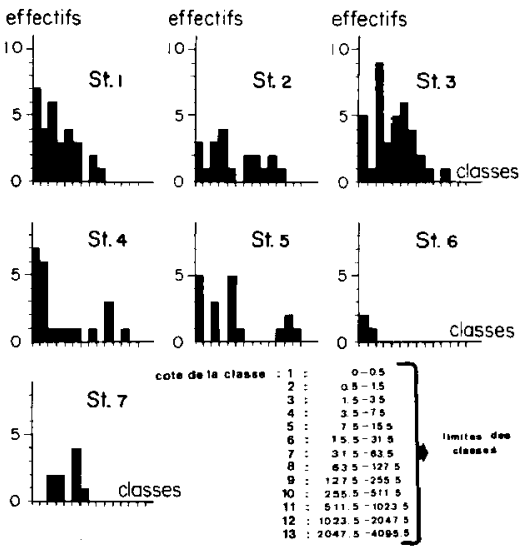

Fig. 11. Histogramme des distributions abondance-especes aux diverses stations. 
la distribution est voisine de celle observée en 1 . La dominance de certains taxa aux stations 4 et 5 provoque un allongement de l'histogramme vers la droite accompagné, surtout en 4, d'un effondrement des classes moyennes qui dominent à nouveau à la station 7.

L'évolution de $\bar{x}$ (fig. 10B) est comparable à celle de $H^{\prime}$ mais en sens inverse sauf en 4 où les taxa à effectifs moyens sont peu représentés. Ceci influence fortement ce paramètre qui traduit la diversité (Bournaud \& Keck 1980). Calculée pour les seuls Oligochètes, $\bar{x}$ traduit l'amélioration en 3 qu'elle ne traduisait pas précédemment lorsqu'elle était calculée sur l'ensemble de la faune (Giani 1983).

$\mathbf{M}_{3}$ croît brutalement à chaque détérioration des conditions de milieu (stations 2,4 et 5 ; il diminue en 3 où la qualité des eaux s'améliore (fig. $10 \mathrm{C}$ ). $\mathbf{M}_{3}$ traduit l'allongement vers la droite de la distribution espèce-abondance et donc la dominance. Appliqué aux seuls Oligochètes, il a autant de signification qu'utilisé sur l'ensemble de la faune (Giani 1983).

\section{4. - Variations saisonnières des indices}

L'indice de diversité $\mathrm{H}^{\prime}$, calculé aux diverses dates de prélèvement (tableau IV) montre que les conditions de milieu sont toujours défavorables au mois d'août, période ou les eaux usées constituent $90 \%$ du débit (S.O.D.E.T.E.G. 1976). Cependant les valeurs minimales peuvent être observées en dehors de la période estivale : octobre et avril en 1, décembre en 3. Les conditions de milieu les plus favorables (tel- les qu'elles sont traduites par cet indice) sont notées en juin aux stations amont (1 et 2), en octobre en 3 et en février en 4 et 5 . Ces valeurs extrêmes sont toujours à mettre en relation avec le cycle biologique de certaines espèces (Naididae en 1,2 et 3) ou avec le débit (en été à toutes les stations, en février en 4 et 5). Le meilleur indice est parfois observé au niveau de stations très polluées : 2 en décembre, 3 en octobre; les valeurs sont même supérieures à 3 ce qui selon Wilhm (1970) traduit une bonne qualite de l'eau. Et, paradoxalement, c'est la station 1, la moins polluée, qui présente l'indice le plus faible en avril.

La même étude, faite avec $M_{3}$ (tableau IV) fournit des résultats aussi incohérents et souvent différents de ceux obtenus avec $H^{\prime}$. Les valeurs ponctuel. les de ces indices sont trop influencées par les variations saisonnières liées soit au cycle biologique soit à l'amélioration des conditions de milieu en fonction du débit et de la température. Comme Ghetti \& Bonazzi (1977) il nous semble nécessaire d'observer les valeurs de ces indices sur un cycle annuel. Cependant, certains indices calculés sur l'ensemble de la faune lorsque les conditions sont les meilleures, rendent compte des variations dans la qualité des eaux conformes à celles observées lors des périodes de pollution maximale (Viaud \& Lavandier 1981).

A titre de comparaison avec ces indices structuraux, nous avons calculé quelques paramètres proposés dans la littérature pour caractériser le milieu à partir des seuls peuplements d'Oligochètes. Ils sont présentés dans les tableaux V et VI et seront discutés au paragraphe suivant.

Tableau IV. Valeurs de l'indice de diversité $\left(\mathrm{H}^{\prime}\right)$ et du moment d'ordre 3 des distributions des cotes d'abondance $\left(\mathbf{M}_{3}\right)$ aux diverses stations à chaque période de prélèvement.

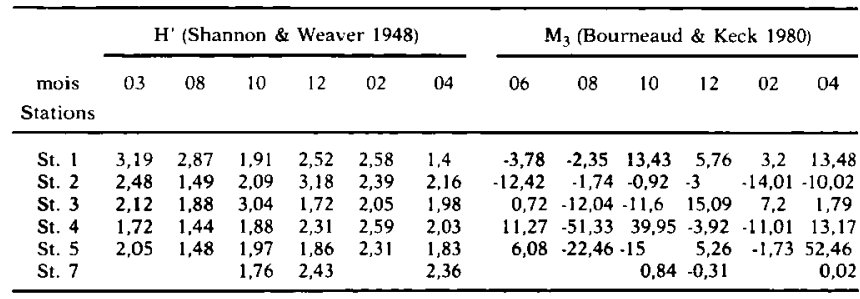


Tableau V. Valeurs, à chacune des stations, de quelques paramètres proposés dans la littéra. ture pour caractériser le milieu à partir des peuplements d'Oligochètes.

\begin{tabular}{cc|c|c|c|c}
\hline & $\begin{array}{c}\text { L. hoffmeisteri } \\
\text { en \% } \\
\text { (Brinkhurst- } \\
1966)\end{array}$ & $\begin{array}{c}\text { L. hoffmeisteri } \\
+ \text { L. udekemia- } \\
\text { nus et T. tubifex } \\
\text { en \% }\end{array}$ & $\begin{array}{c}\text { L. hoffmeisteri } \\
+ \text { L. udekemia- } \\
\text { nus } \\
\text { Stations }\end{array}$ & $\begin{array}{c}\text { Index de } \\
\text { trophie } \\
\text { Milbrink } \\
(1966)\end{array}$ & $\begin{array}{c}\text { Index de } \\
\text { trophie } \\
\text { modifié } \\
\text { (cf texte) }\end{array}$ \\
St. 1 & 4,3 & 5,8 & 7,4 & 1,88 & 2,05 \\
St. 2 & 4,3 & 22,5 & 69,6 & 1,99 & 2,89 \\
St. 3 & 7,3 & 9,5 & 66 & 2,89 & 2,94 \\
St. 4 & 18,6 & 65,1 & 83,7 & 2,39 & 2,88 \\
St. 5 & 22,2 & 56,5 & 71,7 & 2,37 & 2,72 \\
St. 7 & 28,4 & 48 & 62,8 & 2,31 & 2,66 \\
\hline
\end{tabular}

\section{4. - Discussion}

Les communautés d'Oligochètes du Riou Mort sont tout à fait comparables aux données de la littérature (Richardson 1928, Brinkhurst 1962, 1969 etc, Kennedy 1965. Howmiller \& Beeton 1971, Ladle 1971, Learner \& al 1971, Milbrink 1973, Gross 1976, Biesiadka \& Kasprzak 1977, Dratnal \& Kasprzak 1980, Mc Lusky et al 1980 etc...): la pollution tend à restreindre la variété des organismes en mème temps qu'apparaissent ou persistent des formes polluorésistantes. La nouvelle communauté qui s'installe présente une faible diversité et une forte densité. Cette évolution générale est à la base des critères utilisés dans l'étude biologiques des pollutions

Tableau VI. Valeurs, à chacune des stations, des paramètres proposés par Lafont (1982) pour caractériser le milieu à partir des communautés d'Oligochètes $\left(I^{\circ}=\frac{10 S}{T}\right.$ avec : $S$, nombre d'espèces et $T$, abondance des Tubificidae dépourvus de soies capillaires).

\begin{tabular}{crccc}
\hline Paramètres & Io & Eo & $\begin{array}{c}\text { Tubificidae } \\
\text { sans soies } \\
\text { capillaires en \% }\end{array}$ & $\begin{array}{c}\text { Nombre } \\
\text { d'espèces } \\
\text { présentes }\end{array}$ \\
\hline Stations & & & & \\
St.1 & 24,5 & $\mathbf{F}_{16}$ & 12,2 & 30 \\
St. 2 & $\mathbf{6 , 8}$ & $\mathbf{E}_{11}$ & 29,3 & 20 \\
St. 3 & $\mathbf{3 0 , 6}$ & $\mathbf{F}_{18}$ & 11,5 & 35 \\
St. 4 & $\mathbf{2 , 8}$ & $\mathbf{B}_{12}$ & 76,5 & 22 \\
St. 5 & $\mathbf{2 , 1}$ & $\mathbf{B}_{10}$ & $\mathbf{8 3 . 5}$ & 18 \\
St. 7 & 1,1 & $\mathbf{B}_{5}$ & $\mathbf{8 0 , 4}$ & 9 \\
\hline
\end{tabular}

(cf. la révision de Hellawell 1978 et plus particulièrement pour les Oligochètes celles d'Aston 1973 , Goodnight \& Whitley 1961, Brinkhurst 1962, 1966. 1980 et Milbrink 1980).

Le premier classement de qualité des eaux en fonction des Oligochetes est celui de Goodnight \& Whitley (1960) qui est basé sur l'abondance relative des Oligochètes dans le peuplement : moins de $60 \%$, bonnes conditions; 60 à $80 \%$, douteux; plus de $80 \%$, fortement pollué. Cette approche est grossière mais non dénuée de fondement : le Riou Mort en est un exemple (tableau II).

Brinkhurst (1966), remarquant la constance de $T$. tubifex et $L$. hoffmeisteri dans les milieux pollues, a proposé d'utiliser le pourcentage de $L$. hoffmeisteri dans la communauté d'Oligochètes comme indicateur de la qualité des eaux. Rappelons que T. tubifex seule ne peut-être considérée comme une espèce indicatrice (Brinkhurst 1962, 1964, Dzillo 1967, Milbrink 1980). Ce critère ignore la densité du peuplement. Dans certains milieux comme le Riou Mort ou ceux étudiés par Gross (1976), Dumnincka \& Pasternak (1978) et Eyres \& al (1978) L. udekamianus peut supplanter $L$. hoffmeisteri. Le critère peut être établi en prenant en compte simultanément ces deux espèces et il devient alors un bon critère pour caractériser la pollution (tableau $\mathrm{V}$ ).

Gross (1976) a proposé une technique de classification en trois étapes faisant intervenir le pourcentage d'Oligochètes par rapport à l'ensemble de la faune, l'abondance relative de la communauté indicatrice formée de T. tubifex, $L$. hoffmeisteri et $L$. ude kemianus, la diversité et la densité des Oligochètes. 
Toutefois son chiffrage ne prend en compte que les deux premiers critères : \% d'Oligochètes faible, communauté indicatrice non développée : pas de pollution; plus de $50 \%$ d'Oligochètes, communauté indicatrice représentant moins de $50 \%$ des Oligochètes : pollution moyenne; plus de $70 \%$ d'Oligochètes et communauté indicatrice supérieure à $70 \%$ : pollution forte. En outre, l'auteur limite l'utilisation de cette méthode aux substrats argilo-vaseux or, comme nous l'avons constaté dans le Riou Mort à la suite de Lafont (1977) et Dumnicka \& Pasternak (1978), on assiste en milieu forternent pollué à une extension de l'habit at de T. tubifex, L. hoffmeisteri, $L$. udekemianus et $N$. elinguis vers les substrats rocheux et sableux à partir de l'habitat vaseux type. Le principe de cette méthode et notamment le pourcentage de la communauté indicatrice (tableau $V$ ) semble utile dans la hiérarchisation des pollutions caractérisées mais il ne permet pas de détecter les pollutions faibles. Cette détection est certainement possible avec la grille de qualité des eaux proposée par Lafont (1977), basée sur l'abondance relative de $N$. elinguis, $S$. heringianus, $T$. tubifex et les « Tubificidae ". Toutefois $T$. tubifex est mal choisie (voir plus haut) et la notion de Tubificidae mérite d'être précisée: dans la partie haute des rivières Peloscolex velutinus, $T$. ignotus, Peristodrilus montanus et $R h$. coccineus peuvent dominer le peuplement et ce sont des espèces indicatrices d'une bonne qualité des eaux. Une synthèse entre cette grille et la méthode de Gross (1976) pourrait donner de bons résultats.

Lafont (1982) a proposé deux indices pour la classification des sédiments fins des grandes rivières. Ils prennent en compte le nombre d'espèces présentes mais regroupent les Tubificidae dépourvus de soies capillaires alors que Milbrink (1978, 1980) insiste sur la nécessité d'un dénombrement spécifique même chez les immatures. Les valeurs de ces indices aux stations 2 et 3 (tableau VI) ne correspondent pas à la réalité de la pollution à ces localités ; le pourcentage de Tubificidae dépourvus de soies capillaires est très proche des critères précédents basés sur le pourcentage de $L$. hoffmeisteri et/ou $L$. udekemianus et le pourcentage de la communauté indicatrice définie par Gross (1976).

Parallèlement à ces études les Oligochètes ont été utilisés comme indicateurs de trophie dans les lacs (Milbrink 1973, 1978, 1980, 1983 ; Brinkhurst 1974 ; Lang \& Lang-Dobler 1980). Howmiller \& Scott (1977) ont proposé un indice de trophie qui a été modifié par Milbrink (1980, 1983). Celui-ci prend en compte la composition spécifique: les Tubificidae sont répartis en 4 groupes selon leur écologie ; chaque groupe a un poids spécifique différent sur la valeur de l'index. Nous avons calculé cet index pour le Riou Mort (tableau V) selon les données de Milbrink (loc. cit.) et sous une forme légèrement modifiée comptetenu de nos observations: $L$. profundicola et $L$. udekemianus sont placés chacun dans un groupe supérieur à celui attribué par cet auteur. Les valeurs obtenues refletent bien les variations des conditions de milieu mais restent cependant faibles selon nous. Cet index de trophie qui semble très performant pour les lacs pourrait être adapté aux rivières après quelques modifications minimes. Il devrait tenir compte des Naididae d'une part et des densités d'Oligochètes qui peuvent être très supérieures dans les milieux courants. Dans les lass en effet, la quantité d' $\mathrm{O}_{2}$ est fréquemment le facteur qui limite la densité d'Oligochètes. En eau courante en revanche, la quantité d'O $\mathrm{O}_{2}$ est généralement suffisante et nous avons vu qu'en présence de matière organique, des populations très importantes peuvent s'établir même sur substrats rocheux ou sableux en courant, le seul facteur limitant est la compétition spatiale (Caspers 1980). Toutefois, en saison chaude, la chute des teneurs en $0_{2}$ s'accompagne d'une réaction des Oligochètes (devenus en surnombre) qui s'agglomèrent et nous avons pu observer de véritables boules vivantes de vers roulants sur le fond (en août à la station 5) identiques à celles observées dans le port de Toronto par Brinkhurst (in Milbrink 1980).

Dans l'étude du Riou Mort nous avons également calculé quelques indices structuraux couramment utilisés sur l'ensemble des communautés d'invertébrés. Leur application a fait l'objet de nombreux travaux comparatifs et critiques (Hulbert 1971, Olive \& Dambach 1973, Dills \& Rogers 1974, Ghetti \& Bonazzi 1977, Howmiller \& Scott 1977, Nagell \& al 1977, Connel 1978, Hellawell 1978, Bournaud \& Keck 1980, Viaud \& Lavandier 1981, Milbrink 1983). Les résultats essentiellement comparatifs donnent une image très variable de l'évolution des communautés dans les cours d'eau. L'indice de Shannon et Weaver appliqué aux Oligochètes et utilisé sur des valeurs moyennes annuelles, comme nous l'avons vu, reflète assez bien l'évolution des conditions de milieu. Le paramètre $M_{3}$ (Bournaud \& Keck 1980) appliqué dans les mêmes conditions, en tant qu'indice de dominance, est particulièrement adapté 
aux peuplements d'Oligochètes. Mais l'utilité de tels indices dans une ćtude basée sur un seul groupe zoologique ne paraît pas évidente et notre travail montre que des paramètres simples comme :

- l'importance relative des Oligochètes dans la communauté,

- la proportion relative des différentes familles d'Oligochètes,

- les espèces dominantes et les espèces secondaires et leurs caractéristiques écologiques (avec notamment $L$. rivalis dont l'abondance est un bon indicateur de pollution),

- le nombre de taxa,

- et la densité de peuplement reflètent bien l'évolution des conditions de milieu.

L'étude de la seule taxocénose des Oligochètes apporte autant d'information que l'étude du reste de la faune. Avec les Oligochètes, il est préférable d'utiliser la notion de communauté indicatrice plutôt que celle d'espèce indicatrice comme le soulignait Milbrink (1980). Dans le domaine des pollutions fortes, l'étude des Oligochètes présente un avantage incontestable : ils permettent, en toute saison, une hiérarchisation des pollutions en l'absence saisonnière ou définitive d'arthropodes. Une difficulté majeure réside toutefois dans les problèmes de taxonomie. Dans le domaine des pollutions faibles, les Oligochètes sont d'une utilité plus contestable. Peut-être des méthodes basées sur des espèces telles $N$. elinguis, S. heringianus (cf. Lafont 1977) et $L$. profundicola pourraient-elles, à condition d'être affinées, détecter les légères perturbations de milieu. De même, l'index de trophie (Howmiller \& Scott 1977, modifié par Milbrink 1983) pourrait être adapté et utilisé avec de bons résultats. Il est toutefois indéniable que pour détecter et caractériser une pollution faible, l'étude des Ephémèroptères et des Plécoptères est de loin préférable à celle des Oligochètes car, comme le soulignait Brinkhurst (1962), il n'y a pas d'espèces d'Oligochètes dont la présence ou l'absence puisse servir d'indicateu:

\section{Travaux cttés}

Aston (R.J.). 1973. - Tubificids and water quality : a review. Environ. Pollut., 5 : 1-10.

Bennike (S.A.B.) \& Berg (K.). 1948. - Biological studies on the River Susaa (Oligochaeta). Folia limnol scand. 4:40-54.

Biesiadka (E.) \& Kasprzak (K.). 1977. - An investigation on the macrofauna of the river Warta within the city of Poznan. Acta Hydrobiol., $19: 109-122$.
Bournaud (M.) \& Keck (G.). 1980. - Diversité spècifique et structure des peuplements de macro-invertébrés benthiques au long d'un cours d'eau: le Furans (Ain). Acta. Oecologia. Oecol. Gener., $1: 131-150$.

Brinkhurst (R.O.). 1962. - The biology of the Tubificidae with special reference to pollution. Proc. 3rd Seminar Biological Problems in Water Pollution. Cincinnati : 57-65.

Brinkhurst (R.O.). 1964, - Observations on the biology of the Tubificidae (Oljgochaeta). Verh. Intemat. Verein. Limnol.. 15 : 855.863 .

Brinkhurst (R.O.). 1965. - Observations on the recovery of a British river from gross organic pollution. Hydrobiologia, $25: 9-51$.

Brinkhurst (R.O.). 1966. - Detection and assessment of water pollution using Oligochaete worms. Water Sewage Works, 113 : 398-401 (part I), 438-441 (part II).

Brinkhurst (R.O.). 1966. - The Tubificidae (Oligochaeta) of polluted waters. Vehr. Intemal, Verein. Limnol., 16 (2) : 854-859.

Brinkhurst (R.O.) 1967. - The distribution of Aquatic Oligochaetes in Saginaw Bay, Lake Huron. Limnol Oceanogr., 12 : 137-143.

Brinkhurst (R.0.). 1969. - The fauna of pollution, in : * The Great Lakes as an environment $G^{\prime}$. Lakes Inst. Univ. Toronto, P.R. 39.

Brinkhurst (R.O.). 1980. - Pollution biology - the North Amerjcan experience. In Aquatic oligochoete Biology, Brinkhurst (R.O.) \& Cook (D.G.) eds Plenum Press, New-York : 471-475.

Brinkhurst (R.O.) \& Kennedy (C.R.). 1965. - Studies on the biology of the Tubificidae in a polluted stream. J. Anim. Ecol., 34: 429.443.

Caspers (H.). 1980. - The relationship of saprobial conditions to massive populations of tubificids. In Aquaric Oligochaele Biology, Brinkhurst (R.O.) \& Cook (D.G.) éds, Plenum Press, NewYork : 503.505 .

Chapman (P.M.), Churchland (L.M.), Thomson (P.A.) \& Michnowsky (E.). 1980. - Heavy metal studies with Oligochaetes. In Aquatic Oligochaete Biology, Brinkhurst (R.O.) \& Cook (D.G.) éds., Plenum Press, New-York : $\mathbf{4 7 7 . 5 0 2 .}$

Connell (J.H.). 1978, - Diversity in tropical rain forests and coral reefs. Science, 199: 1302-1310.

Dills (G.) Rogers (D.T.Jr). 1974, - Macroinvertebrate community structure as an indicator of acid mine pollution. Environ. Pollut., $6: 239.262$.

Dratnal (E.) \& Kasprzak (K.). 1980 - The response of the invertebrate fauna to organic pollution in a well oxigenated karst stream exemplified by the Pradnik Stream (South Poland). Acta Hydrobiol, 22 : 263-278.

Dumnicka (E.) 1978 - Communities of oligochaetes (Oligochaeta) of the river Nida and its tributaries. Acta Hydrobiol, 20: 117-141.

Dumnicka (E.) \& Pasternak (K.). 1978, - The influence of physicochemical properties of water and bot tom sediments in the river Nida on the distribution and numbers of Oligochaeta. Acta Hydrobiol. 20 : 215-232.

Dzwillo (M.\}.1967 - Untersuchungen über die Zusammensetzung der Tubificidenfauna im Bereich des Harmburger Hafens, Vehr. Norurw. Ver. Hamburg., 11 : 101-116.

Eyres (J.P.), Williams (N.V.) \& Pugh-Thomas (M.). 1978. Ecological studies on Oligochaeta inhabiting depositing substrata in the Irwell, a polluted English river. Freshw. Biol., 8 : 25-32.

Ghetti (F.) \& Bonazzi (G.). 1978. - A comparison between various criteria for the interpretation of biological data in the analysis of the quality of running waters. Wat. Res., $11: 819-831$.

Giani (N.). 1983. - Le Riou Mort, affluent du Lot pollué par métaux lourds. III. Etude faunistique générale. Annals Limnol. $19: 29-43$.

Giani (N.) \& Martinez-Ansemil (E.) + 1981 _ Cont ribution à la connaissance des Oligochètes aquatiques du bassin de l'Argens (Nar. France). Annis Limnol., $17: 121-141$. 
Goodnight (C.J.) \& Whitley (L.S.) 1960 - Oligochaetes as indi. cators of pollution. Wat. Sewage Wks, 107.311.

Hawkes (H.A.). 1962. - Biological aspects of river pollution, $p p$ 311.342. In L. Klein, River pollution, Butterworth, London : 456 p.

Hellawel] (J.M.), 1978 - Biological surveillance of rivers. Water Research centre : Stevenage Laboratory. England.

Howmiller (R.P.) et Beeton (A.M.). 1970. - Biological evaluation of enviromental quality, Green Bay, Lake Michigan. 1. Waser Pollut. Control. Fed, $43: 123+133$.

Howmiller (R.P.) \& Scott (M.A.). 1977. - An environmental index based on relative abundance of oligochaete species. J. Water. Control. Fed. $49: 809-815$.

Hulbert (S.H.) 1971. - The nonconcept of species diversity : a critique and alternative parameters. Ecology. 52:577-586.

Juget (J.) \& Giani (N.). 1974. - Répartition des Oligochètes lacus tres du Massif de Neouvielle (Hautes.Py rénées) avec la descrip tion de Peloscolex pyrenaicus n. sp. Annls Limnol.. 10 : $33-53$.

Kasprzak (K.). 1971. - Contribution to the knowledge of Oligo chaeta of Poznan. Bad. Fizjogr. Pol. Zach. Biol., 24 : 225-229.

Kasprzak (K.). 1976. - Investigations of Oligochaeta of the lower part of Welna River (Poland). Fragm. Faun., 20: $425-467$

Kennedy (C.R.). 1965. - The distribution and habitat of Limnodrilus Claparède (Oligochaeta, Tubificidae). Oikos, $16: 26-38$.

Ladle (M.). 1971. - The Biology of Oligochaeta from Dorset chalk streams. Freshw, Biol, $1: 87.97$.

Lafont (M.). 1977a. - Les Oligochètes d'un cours d'eau montagnard pollué : le Bief Rouge. Annls Limnol, 13 : 157.167.

Lafont (M.). 1977b. - Les oligochètes et la détection des pollutions dans les cours d'eau. L'eau et l'industrie, $17: 84-85$.

Lafont (M.). 1982 - Oligochaete communities as biological descriptors of pollution in the fine sediments of rivers. Second International Symposium on Aquatic Oligochaeta Biology, Pallanza, Italie (sous presse).

Lafont (M.) \& Juget (J.). 1981. - Les Oligochètes de quelques lacs jurassiens et leur utilisation pour apprécier l'êtat biologique des sédiments profonds. Arrnls Sci. Univ. Besançon, 2 : 47.57.

Lang (C.) \& Lang-Dobler (B.). 1980. - St nucture of tubificid and Lumbriculid worm communities, and three indices of trophy basec upon these communities, as descriptors of eutrophication level of Lake Geneva (Switzerland). In Aquatic Oligochaeta Biology. Brinkhurst (R.O.) et Cook (D.G.) eds. Plenum Press, New-York : $467-470$.

Leamer (M.A.). 1979. - The distribution and ecology of the Naididae (Oligochaeta) which inhabit the filter-beds of sewageworks in Britain. Water Res., 13: 1291-1299.

Learner (M.A.). Williams (R.), Harcup (M.) \& Hughes (B.D.). 1971. - A survey of the macrofauna of the river Cynon, a polluted tributary of the river Taff (South Wales). Freshw. Biol., $1: 339.368$.

Learner (M.A.), Lochhead (G.) \& Hughes (B.D.). 1978. - A review of the biology of British Naididae (Oligochaeta) with emphasis on the lotic environment. Freshw. Biol., $8: 357-375$.

Martinez-Ansemil (E.). 1982. - Les Oligochètes aquatiques de la Péninsule Ibèrique (2e note), avec la description de Lumbricitlus brunoi n. sp. (Enchytraeidae). Bull. Soc Hist. Nat. Toulouse, 118: 145-151.

Mc Lusky (D.S.), Teare (M.) \& Phizacklea (P.). 1980. - Effects of domestic and industrial pollution on distribution and abundance of aquatic oligochaetes in the Forth estuary. Helgol. Meeres., $33: 384-392$.
Milbrink (G.) 1973 . - On the use of indicator communities of Tubificidae and some Lumbriculidae in the assessment of Water pollution in the Swedish Lakes. Oikos, 1 : 125-139.

Milbrink (G.). 1978. - Indicator communities of Oligochaetes in Scandinavian Lakes. Vehr. Intemat. Verein, Limnol, 20 : $2406-2411$.

Milbrink (G.). 1980. - Oligochaete communities in pollution biology. The Europacan situation with special references to lakes in Scandinavia. In Aquatic Oligochaera Biology. Brinkhurst (R.O.) et Cook (D.G.) éds. Plenum Press, New-York : 433-455.

Milbrink (G.) 1983. - An improved environmental index based on the relative abundance of oligochaete species. Hydrobiolo gia, 102:89-97.

Nagell (B.), Landahl (C.C.) et Lann (H.). 1977, - Ouantitative and qualitative composition of bottom fauna in polluted parts of Lake Vänem and some aspects on water quality indices. Vatten $4: 434-441$.

Nut tal (P.M.). 1973. - The effects of refuse-tip liquor upon stream biology. Environ. Pollut, $4: 215-222$.

Olive (J.H.) \& Dambach (C.A.). 1973. - Benthic macroinvertebrates as indexes of water quality in Wheststone Creek, Morrow County, Ohio (Scioto River Bas in). Ohio J. Sci., $73: 129.149$.

Pielou (E.C.). 1975, - Ecologicol diversity. Chichester, $176 \mathrm{p}$.

Richardson (R.E.), 1928. - The bottom fauna of the middle Illinois river, 1913-1925. Bull. IIl. Lab. Nat. Hist, $17: 387-475$.

Say (P.J.). 1978. - Le Riou Mort, affluent du Lot pollué par métaux lourds. I. Etude préliminaire de la chimie et des algues benthiques. Annls Limnol., 14: 113-131.

Say (P.J.) \& Giani (N.). 1918. - The Riou Mort, a tributary to the river lot polluted by heavy metals. Il. Accumulation of zinc by Oligochaetes and Chironomids. Acta Oecologia, Oecol Applic., 2: 339-355.

Shannon (C.E.) \& Weaver (W.). 1948. - A mathematical theory of communication. Bell. Syst. Techn. J., $27: 379-423,623-656$.

Simpson (E.H.). 1949. - Measurement of diversity. Nature, 163 : 668.

S.O.D.E.T.E.G. 1976. - Présentation et symthèse des études menées sur la vallee du Lot dans le cadre de l'opération * Lot, rivière claire ". Toulouse, $247 \mathrm{p}$.

Szczesny (B.). 1974, - The effect of sewage from the town of Krynica on the benthic invertebrates communities of the Kryniczanka stream. Acta Hydrobiol, 16 : 1.29.

Viaud (M.) \& Lavandier (P.). 1981 - Comparaison de quelques indices usuels d'évaluation de la qualité des eaux appliqués à la détection de pollutions faibles. Bull. Sor. Hist. Nat., Toulouse, $117: 221 \cdot 230$.

Wilhm (J.L.) 1970 - Range of diversity index in benthic macroinvertebrate populations. J. Water Poll Contr. Fed., $42: 221-224$.

Williams (N.V.) \& Taylor (H.M.). 1968. - The effect of Psychoda alternata (Say.) (Diptera) and Lumbricillis rivalis (Levinsen) (Enchytraeidae) on the efficiency of sewage treatment in percolating filters. Waters Res., $2: 139-150$.

Williams (N.V.). Solbé (J.F. de L.G.) \& Edwards (R.W.). 1969. Aspects of the distribution, life history and metabolism of the Enchytraeid worms Lumbricillus rivalis (Levinsen) and Enchytraeus coronatus $(\mathrm{N} \& \mathrm{G}$ ) in a percolating filter. J. Appl. Ecol., $6: 171-183$.

Woodiwiss (F.S.). 1964. - The biological system of stream classification used by the Trent River Board. Chem. Ind., 11: 443-447. 